Goll, Annalena; Kopmann, Rebekka; Baron, Manuela

Kontinuierliche Validierung hydrodynamischer und morphodynamischer Modelle mit physikalischen Modellversuchen

Verfügbar unter / Available at:

https://hdl.handle.net/20.500.11970/100669

Vorgeschlagene Zitierweise / Suggested citation:

Goll, Annalena; Kopmann, Rebekka; Baron, Manuela (2013): Kontinuierliche Validierung hydrodynamischer und morphodynamischer Modelle mit physikalischen Modellversuchen. In: Wasserwirtschaft Jg. 103 (12/2013). S. 28-33. 


\title{
Autorenfassung
}

Goll, Kopmann, Baron: Kontinuierliche Validierung hydrodynamischer und morphodynamischer Modelle mit physikalischen Modellversuchen, 2013

Erstveröffentlichung in WasserWirtschaft 12 (2013), S. 28-33.

Für eine korrekte Zitierbarkeit ist die Seitennummerierung der Originalveröffentlichung für jede Seite kenntlich gemacht.

\section{Kontinuierliche Validierung hydrodynamischer und morpho- dynamischer Modelle mit physikalischen Modellversuchen}

\author{
Annalena Goll, Rebekka Kopmann und Manuela Baron
}

\section{Einleitung}

Mehrdimensionalenumerische Verfahren zur Berechnung von Fließgewässerströmungen, wie die Telemac-Suite (www.opentelemac.org), haben bereits einen beachtlichen Umfang erreicht und finden zunehmend Anwendung in der wasserbaulichen Praxis. Dabei spielt durch die ökologische Sensibilisierung in den letzten Jahrzehnten die morphodynamische Gewässerentwicklung inzwischen eine erhebliche Rolle. In der Bundesanstalt für Wasserbau (BAW) gehören mehrdimensionale numerische Feststofftransportmodelle zur Lösung von instationären, großskaligen Problemen inzwischen zum Standard.

Die dezentrale Weiterentwicklung der numerischen Software liefert dem Anwender mittlerweile in kurzen Zeitabständen neue Module und Lösungsmethoden. Allerdings setzt eine erfolgreiche Anwendung dieser Verfahren ein grundlegendes Verständnis der physikalischen Phänomene sowie Erfahrung in der numerischen Modellierung voraus. Dies ist bedingt durch die Vielfalt und Komplexität der morphodynamischen Phänomene (insbesondere durch Rückkopplungsprozesse zwischen Strömung und der sich umlagernden Sohle) sowie durch deren Beschreibung mittels semiempirischer Gleichungen. Die Zuverlässigkeit dieser Verfahrensklasse kann nur unzureichend durch analytische Lösungen nachgewiesen werden. Regelmäßige Verifikation, Validierung durch Labormodelle sowie die Steigerung des Verständnisses von Modellierern und Entwicklern sind daher maßgebend für die Prognosefähigkeit.

Die BAW betreibt zur Validierung der numerischen Verfahren, u. a. der kontinuierlich wachsenden Telemac-Suite, physikalische Labormodelle mit und ohne bewegliche Sohle, in denen verschiedene Phänomene isoliert betrachtet werden können. Im Folgenden sollen zwei Labormodelle kurz vorgestellt werden. Ersteres dient der Untersuchung einer Anwendung für die flussbauliche Praxis ohne Berücksichtigung der morphodynamischen Änderungen. Das zweite Modell behandelt Dünenbildung sowie -bewegung und gehört in den Bereich der Forschung; es dient der Weiterentwicklung von Labormodellen und der numerischen Simulation. 


\section{Autorenfassung}

Goll, Kopmann, Baron: Kontinuierliche Validierung hydrodynamischer und morphodynamischer Modelle mit physikalischen Modellversuchen, 2013

\section{Hydrodynamische Validierung}

Ein Basisfall der Validierung ist der Vergleich des vertikalen und horizontalen Geschwindigkeitsprofils in einer sehr langen, geraden Rinne mit fester Sohle. Darauf aufbauend können kompliziertere Geometrien, wie z. B. verschiedene Buhnenanordnungen, untersucht werden. Ein Buhnenneubau bed eutet einen starken Eingriff in das Strömungsverhalten eines Flusses. Änderungen der Buhnenlängen oder -höhen habengeringere Auswirkungen. Ein für diese Untersuchungen geeignetes numerisches Modell sollte möglichstgut in der Lage sein sowohl die erheblichen Änderungen infolge Buhnenneubaus als auch die weniger drastischen Folgen durch Buhnenänderungen vorherzusagen. Eine hydrodynamische Validierung von
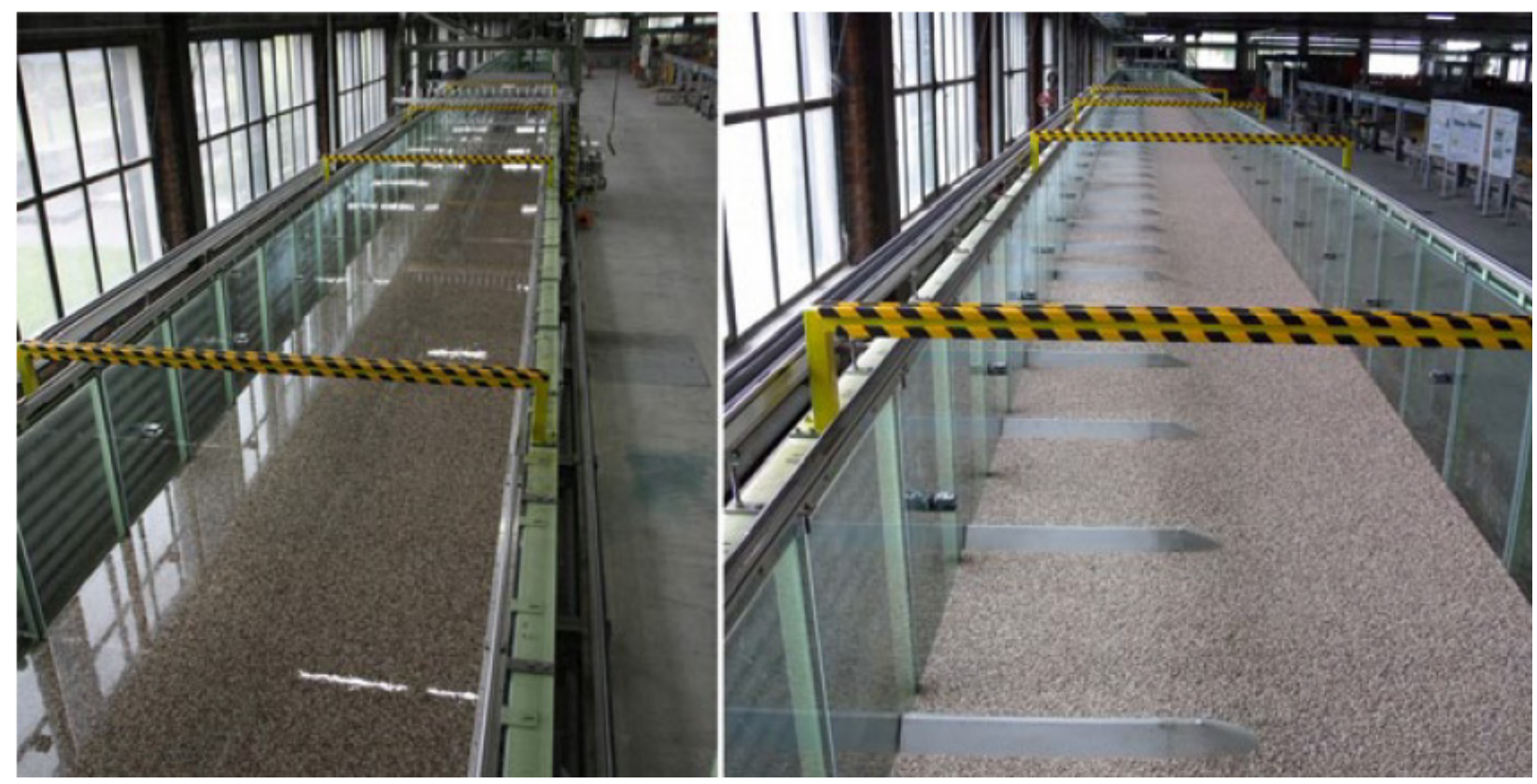

Bild 1: $\quad$ Unverbaute Rinne (links) und Rinne mit Buhneneinbauten (rechts) 


\section{Autorenfassung}

Goll, Kopmann, Baron: Kontinuierliche Validierung hydrodynamischer und morphodynamischer Modelle mit physikalischen Modellversuchen, 2013

Goll, Kopmann, Baron: Kontinuierliche Validierung hydrodynamischer und morphodynamischer Modelle mit physikalischen Modellversuchen. Wasserwirtschaft 12 (2013), S. 28-33.

\begin{tabular}{|c|c|c|c|c|c|}
\hline \multirow[t]{3}{*}{ Verfahren } & \multirow[t]{3}{*}{ Turbulenzmodell } & \multirow[t]{3}{*}{ Advektionsverfahren } & \multicolumn{3}{|c|}{ Wassertiefe im Versuch } \\
\hline & & & $70 \mathrm{~mm}$ & $120 \mathrm{~mm}$ & $160 \mathrm{~mm}$ \\
\hline & & & \multicolumn{3}{|c|}{ Abweichung des WSP-Gefälles [\%] } \\
\hline \multirow[t]{6}{*}{ Telemac- 2D } & \multirow{2}{*}{$\begin{array}{l}\text { Konst. Wirbel- } \\
\text { viskosität }\end{array}$} & Charakteristiken & -2 & -1 & -2 \\
\hline & & MURD & -1 & 0 & -2 \\
\hline & \multirow[t]{2}{*}{ K- $\varepsilon$} & Charakteristiken & -1 & -1 & -1 \\
\hline & & MURD & 0 & 0 & 0 \\
\hline & \multirow[t]{2}{*}{ Elder } & Charakteristiken & -1 & -1 & Abbruch \\
\hline & & MURD & 0 & 0 & 0 \\
\hline \multirow[t]{4}{*}{ Telemac- 3D } & \multirow[t]{2}{*}{ К- $\varepsilon$} & Charakteristiken & 0 & 0 & 0 \\
\hline & & MURD & 0 & 0 & 0 \\
\hline & \multirow{2}{*}{$\begin{array}{l}\text { Hor. Konst. Wir- } \\
\text { belviskosität } \\
\text { Mischungsweg }\end{array}$} & Charakteristiken & -6 & -2 & $-0,5$ \\
\hline & & MURD & -6 & -2 & $-0,5$ \\
\hline
\end{tabular}

Tab. 1: $\quad$ Abweichung des Wasserspiegelgefälles zwischen Messung und Rechnung für die Ausgangsvariante

\subsection{Modellbeschreibung}

\section{Versuchsrinne}

Die Versuche wurden in einer $70 \mathrm{~m}$ langen und 2,5 m breiten Rinne mit einer geneigten, unbeweglichen Kiessohle mit einem Gefälle von 0,6\%o (0,6 mm/m) durchgeführt (Bild 1). Die effektive Rinnenlänge beträgt 62,9 m und die Korngröße des Sohlenmaterials liegt zwischen 4 und $8 \mathrm{~mm}$. Für die Buhnenvariante wurden einseitig 40 Regelbuhnen im Abstand von 1,5 m und mit einem Verhältnis zwischen Buhnenabstand und Buhnenlänge von 1,5 orthogonal zur Strömung sowie mit 


\section{Autorenfassung}

Goll, Kopmann, Baron: Kontinuierliche Validierung hydrodynamischer und morphodynamischer Modelle mit physikalischen Modellversuchen, 2013

einer Höhe von $8 \mathrm{~cm}$ parallel zur Kiessohle eingebaut. Die gewählten Abmessungen wurden typischen Verhältnissen an Bundeswasserstraßen in einem Maßstab von 1:100 angelehnt.

Vertikale und horizontale Geschwindigkeitsprofile wurden mit einem Akustik Doppler (Nortek Vectrino, down- und sidelooking) oder magnetisch-induktiven Sonden (Deltares E-PMS E30) aufgenommen. Darüber hinaus konnten mit einer 3-D-Partikel-Tracking-Velocimetry (3D-PTV) photogrammetrisch horizontale Oberflächengeschwindigkeiten und Wasserspiegel ermittelt werden [2]. Wasserspiegel wurden zusätzlich kontinuierlich mit 18 Wasserspiegel-Messtöpfen gemessen. Detaillierte Beschreibungen des Rinnenversuches finden sich in [3].

\section{Numerisches Modell}

Ausgangs- und Buhnenvariante wurden im Labormaßstab sowohl mit Telemac-2D als auch mit Telemac-3D in der Version V6P1 berechnet. Für die horizontale Auflösung wurden ca. 54000 Knoten mit Kantenlängen zwischen 5 und $10 \mathrm{~cm}$ verwendet. Die Wassertiefe wurde mit zehn logarithmisch, bodennah verfeinerten Schichten diskretisiert. Als Rauheitsgesetz wurde Nikuradsegewählt und es wurden verschiedene Turbulenzmodelle, sowie Advektionsverfahren getestet.

\subsection{Ergebnisse}

Für die unverbaute Rinne sollte das Wasserspiegelgefälle bei verschiedenen Abflüssen mit unveränderlichen Rauheitsbeiwerten für Sohle und Wände abgebildet werden können. Für Telemac-2D ergibt sich daraus eine äquivalente Rauheitshöhe nach Nikuradse von $9 \mathrm{~mm}$ für die Bodenrauheit und $1 \mathrm{~mm}$ für die Wandrauheit. In Telemac-3D wurde bei Verwendung des k-ع-Modells ein Rauheitsbeiwertvon $21 \mathrm{~mm}$ für den Boden verwendet, mit dem Mischungswegmodell nur $6 \mathrm{~mm}$. Tabelle 1 vergleicht die Wasserspiegelgefälle zwischen Messung und Rechnung für 3 verschiedene Versuche mit 70, 120 und $160 \mathrm{~mm}$ Wassertiefe. Sowohl für die 2-D- als auch für die 3-DBerechnungen kann mit dem k- $\varepsilon$-Modell das Wasserspiegelgefälle sehr gut abgebildet werden. Das explizite Advektionsverfahren MURD-Schema (Multidimensional Upwind Residual Distribution) liefert bei den 2-D-Berechnungen leichtbessere Ergebnisse. Bei dem Vergleich der Differenzen der Wasserspiegelgefälle der Buhnenvariante zeigen sich die Vorteile des k- $\varepsilon$-Modells noch stärker [4]. 


\section{Autorenfassung}

Goll, Kopmann, Baron: Kontinuierliche Validierung hydrodynamischer und morphodynamischer Modelle mit physikalischen Modellversuchen, 2013

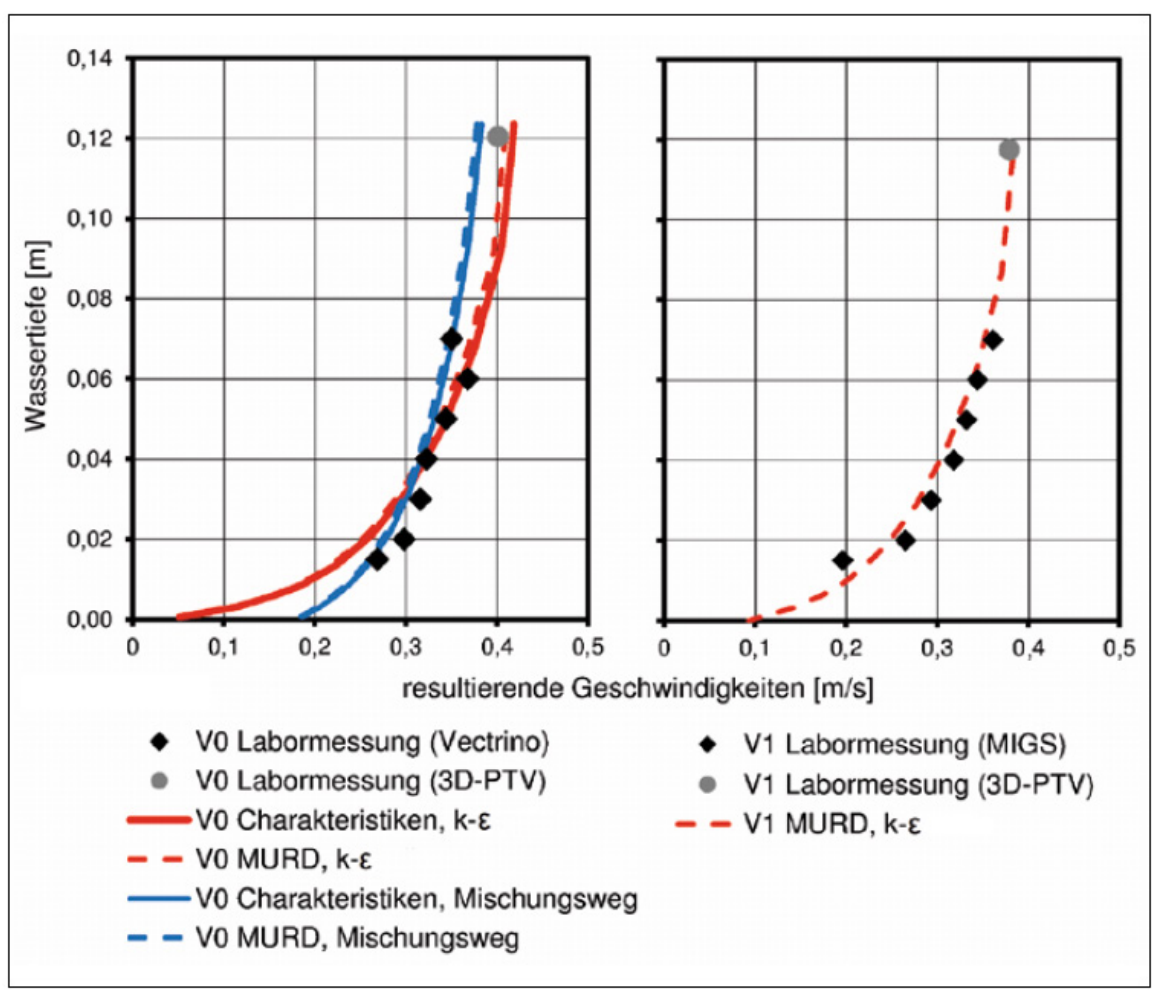

Bild 2: $\quad$ Vergleich des gemessenen und mit Telemac-3D berechneten Geschwindigkeitsprofils an Profil $60 \mathrm{~m}$ für den Wasserstand 120 mm für Ausgangs- und Buhnenvariante

Goll, Kopmann, Baron: Kontinuierliche Validierung hydrodynamischer und morphodynamischer Modelle mit physikalischen Modellversuchen. Wasserwirtschaft 12 (2013), S. 28-33.

Bild 2 zeigt den Vergleich des gemessenen vertikalen Geschwindigkeitsprofils für Profil $60 \mathrm{~m}$ für einen Wasserstand von $120 \mathrm{~mm}$ mit den Ergebnissen von Telemac-3D. Das Turbulenzmodell hat offensichtlich einen großen Einfluss auf die Ausprägung des vertikalen Geschwindigkeitsprofils. Der Prandtl'sche Mischungswegansatz zeigt im bodennahen Profil Vorteile, wohingegen das oberflächennahe Profil mit dem k- $\varepsilon$-Modell besser getroffen wird. Die Unterschiede zwischen den Advektionsverfahren-Charakteristiken und dem explizitem MURD-Verfahren sind vergleichsweise gering.

Mit den in der Ausgangsvariante als optimal gefundenen Einstellungen (k- $\varepsilon$-Modell, MURD-Schema, Bodenrauheitswert von 9 mm für 2D bzw. 21 mm für 3D) wurden Wasserstände und Geschwindigkeiten in der Buhnenvariante simuliert, ohne weitere Parameter anzupassen. Die prozentualen Abweichungen des Wasserspiegelgefälles zwischen Messung und Rechnung lagen unterhalb von 0,1\%o. Das berechnete Geschwindigkeitsprofil in der Hauptströmung bei Profil $60 \mathrm{~m}$ (Bild 2) zeigt eine mit der Ausgangsvariante vergleichbar gute Abbildung des gemessenen Profils. 


\section{Autorenfassung}

Goll, Kopmann, Baron: Kontinuierliche Validierung hydrodynamischer und morphodynamischer Modelle mit physikalischen Modellversuchen, 2013

Bild 3 zeigt den Vergleich der mit 3-D-PTV gemessen Oberflächengeschwindigkeiten im Vergleich zu den Simulationen. Mit Telemac-3D können die Oberflächengeschwindigkeiten direkt erzeugt werden, mit Telemac-2D werden die tiefengemittelten Geschwindigkeiten um einen Faktor 1,3 erhöht, der sich aus der Auswertung der Geschwindigkeitsprofile ergibt, um die Oberflächengeschwindigkeiten zu erhalten.

Die Hauptströmung sowie Form und Intensität der Ablösezone werden sowohl mit Telemac-2D als auch mit Telemac-3D sehr gut getroffen. Lediglich in der Ausbildung des Buhnenwirbels sind Vorteile der 3-D-Berechnungzu sehen. Für beide Verfahren gilt, dass der Buhnenwirbel etwas zu langsam ist.

\subsection{Ausblick}

Die in der unverbauten Rinne gefundenen Einstellungen für 2-D- und 3-D-Berechnungen zeigen eine gute bis sehr gute Abbildung der Strömungssituation der Buhnenvariante. Weitere Buhnenund Bauwerksvarianten wurden bereits untersucht [4] bzw. sind in Planung. Diese Validierungen bestätigen, dass Telemac-2D und Telemac-3D für die Untersuchungen von Bauwerksvarianten geeignet sind. Sie zeigen aber auch, dass die Wahl von Turbulenzmodell sowie Advektionsverfahren einen erheblichen Einfluss auf die Qualität und damit die Prognosesicherheit hat. Die hier gefundenen Aussagen über Turbulenzmodelle müssen im Naturmaßstab bestätigt werden, da die Turbulenzkaskade im Labormaßstab eingeschränkt ist und damit die Übertragbarkeit nicht zwingend vorausgesetzt werden kann. Dazu dienen z. B. aktuelle Untersuchungen der BAW eines gut vermessenen Wirbels in einer Hafeneinfahrt am Elbe-Seitenkanal.

\section{Morphodynamische Validierung}

Dünen sind die häufigsten Sohlenformen der Wasserstraßen. Morphodynamische Berechnung von Fließgewässern verwenden auf Grund der Rechenzeiten noch immer standardmäßig hydrodynamische 2-D-Simulationen als Grundlage. Wenn in diesen Modellierungen ein Düneneinfluss berücksichtigt werden soll, wird meist mittels Dünenlänge und -höhe eine angepasste Sohlenrauheit berechnet, um damit den Einfluss der Transportkörper auf die tiefengemittelte Strömung abzubilden. Somit kommt eine globale und zeitlich nicht variable Sohlenrauheit zum Einsatz. Dies entspricht jedoch nicht der höchst dynamischen Natur der Dünenfelder, die mit Abfluss und anderen externen Einflüssen in Höhe und Länge variieren. Ziel der Untersuchungen der BAW ist daher, Dünen direkt in morphodynamischen Simulationen abzubilden und so mögliche, sich entwickelnde Untiefen und Unterhaltungsmaßnahmen besser abschätzen zu können. In einem Labormodell der BAW finden Dünenexperimente statt, die mit der Telemac-Suite simuliert werden. Der Erkenntnisgewinn aus diesen Simulationen soll Eingang in die Projektarbeit finden.

\subsection{Modellbeschreibung}

\section{Versuchsrinne}




\section{Autorenfassung}

Goll, Kopmann, Baron: Kontinuierliche Validierung hydrodynamischer und morphodynamischer Modelle mit physikalischen Modellversuchen, 2013

Die hier vorgestellte morphologische Versuchsrinne der BAW Karlsruhe(Bild 4) hat eine Länge von $32 \mathrm{~m}$ und eine Gesamtbreite von $5 \mathrm{~m}$. Auf einer Breite von $2 \mathrm{~m}$ wurden Versuche mit bewegten Dünen durchgeführt, die auf einer Länge von $28 \mathrm{~m}$ ausgewertet werden konnten. Die Rinne besitzt einen rechteckigen Querschnitt, eine Seitenwand besteht aus Plastikmaterial, die zweite aus Glas mit metallenen Streben. Der Boden ist zu jeder Zeit mit Sediment bedeckt. Das Sediment ist

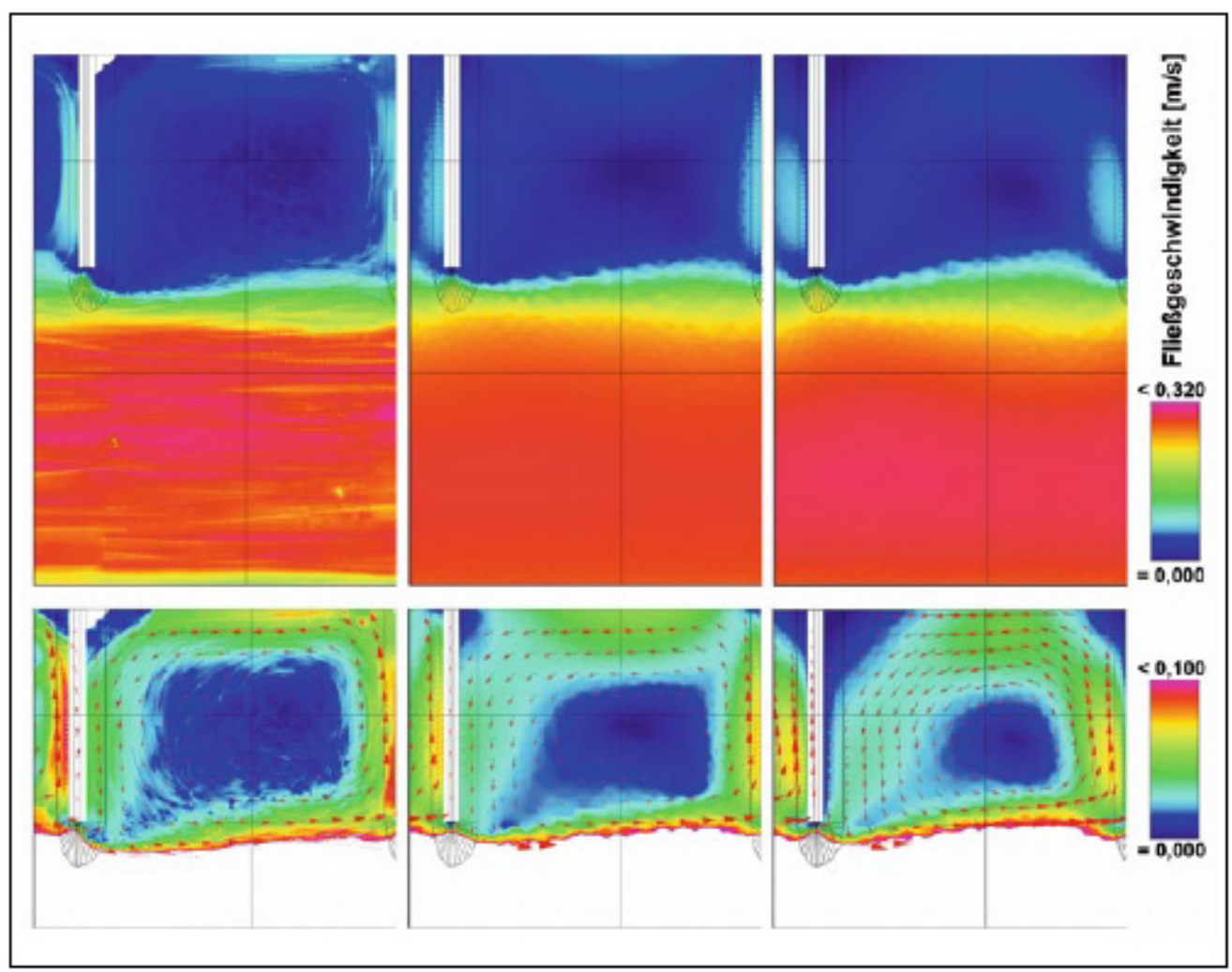

Bild 3: $\quad$ Oberflächengeschwindigkeiten für umströmte Buhnen(Wassertiefe 70 mm) im Buhnenfeld und Hauptströmung(oben) und als Detail im Buhnenfeld (unten) gemessen mit 3-D-PTV (links), berechnet mit Telemac-3D (Mitte) und berechnet mit Telemac-2D (rechts) [4]

Goll, Kopmann, Baron: Kontinuierliche Validierung hydrodynamischer und morphodynamischer Modelle mit physikalischen Modellversuchen. Wasserwirtschaft 12 (2013), S. 28-33.

quasi Einkornmaterial: es besitzt eine sehr steile Sieblinie mit vier Fraktionen mit den jeweiligen mittleren Durchmessern von 0,4275/0,605/0,855/1,2 mm mit einer Verteilung von 1/3/64/32 \%. Der $\mathrm{d}_{50}$ beträgt $94 \mathrm{~mm}$. Der Boden der Rinne wird vor Versuchsbeginn auf ein Gefälle von 0,6\%o $(0,6 \mathrm{~mm} / \mathrm{m})$ gebracht. 


\section{Autorenfassung}

Goll, Kopmann, Baron: Kontinuierliche Validierung hydrodynamischer und morphodynamischer Modelle mit physikalischen Modellversuchen, 2013

Ein regulierbares Wehram Ausfluss bestimmt die Wassertiefe, die anhand von 15 Wasserspiegeltöpfen entlang der Rinne während der gesamten Versuchsdauer aufgezeichnet wird. Sediment wird stündlich, schubweise immer abwechselnd rechts und links im Rinneneinlaufbereich zugegeben. Für die hier beschriebenen Versuche werden ein Abfluss von 145 l/s und eine Sedimentzugabe von $37 \mathrm{~kg} / \mathrm{h}$ verwendet. Hierbei beträgt die Wassertiefe am Auslauf 0,1511 m. Die hier präsentierten Versuche haben eine Versuchsdauer von $18 \mathrm{~h}$.

Mittels eines im Auftrag der BAW entwickelten 3-D-PTV-Messsystems wird die Sohlenoberfläche kontinuierlich durch die Wasseroberfläche hindurch, aufgezeichnet [2].

\section{Numerisches Modell}

Die numerische Simulation des Rinnenversuches wurde mit der Telemac-Suite durchgeführt. Hierbei kamen Telemac- 3D, Sisyphe und DredgeSim in der Version V6P1 zum Einsatz [1], [5], [6].

Das Berechnungsnetz erfasst die komplette Rinnebis auf Einlauf- und Auslaufbereich (Rinnenmeter 2 bis 29,97 in Längsrichtung und über die komplette Breite von $2 \mathrm{~m}$ ). Das unstrukturierte Sigma-Netz hat einen horizontalen Knotenabstand von 5 bis $10 \mathrm{~cm}$. In der Vertikalen sind zehn Ebenen logarithmisch, bodennah verfeinernd, verteilt. Damit ergeben sich 5752 Knoten (10 916 Elemente) im 2-D- und 57520 Knoten im 3-D-Gitter. Die Rechenzeiten liegen für eine 18-stündige Simulation bei $3 \mathrm{~h}$ auf 48 Prozessoren des Großrechners der BAW.

Parameterstudien [7] haben gezeigt, dass die Turbulenzmodellierung für die Simulation von Sohlenformen von immenser Bedeutung ist. Telemac-3D bietet die Wahl zwischen vier verschiedenen Turbulenzansätzen: Zwei algebraische Modell (Mischungswegmodell, z. B. von Prandtl,

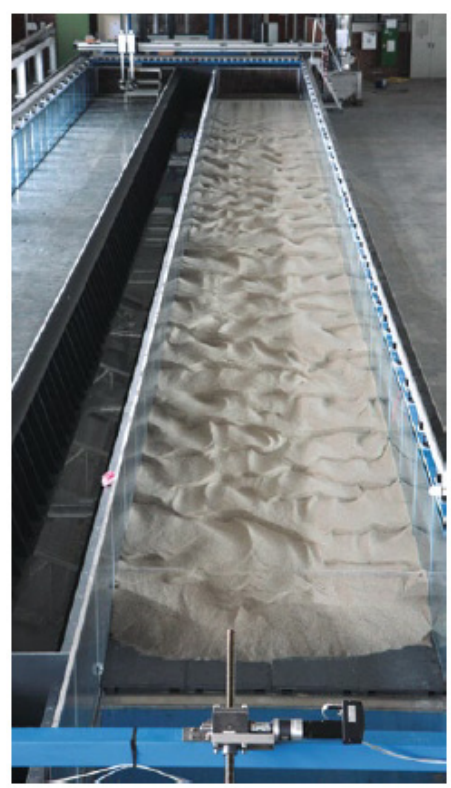

Bild 4: $\quad$ Morphologische Versuchsrinne der BAW Karlsruhe 


\section{Autorenfassung}

Goll, Kopmann, Baron: Kontinuierliche Validierung hydrodynamischer und morphodynamischer Modelle mit physikalischen Modellversuchen, 2013

Goll, Kopmann, Baron: Kontinuierliche Validierung hydrodynamischer und morphodynamischer Modelle mit physikalischen Modellversuchen. Wasserwirtschaft 12 (2013), S. 28-33.

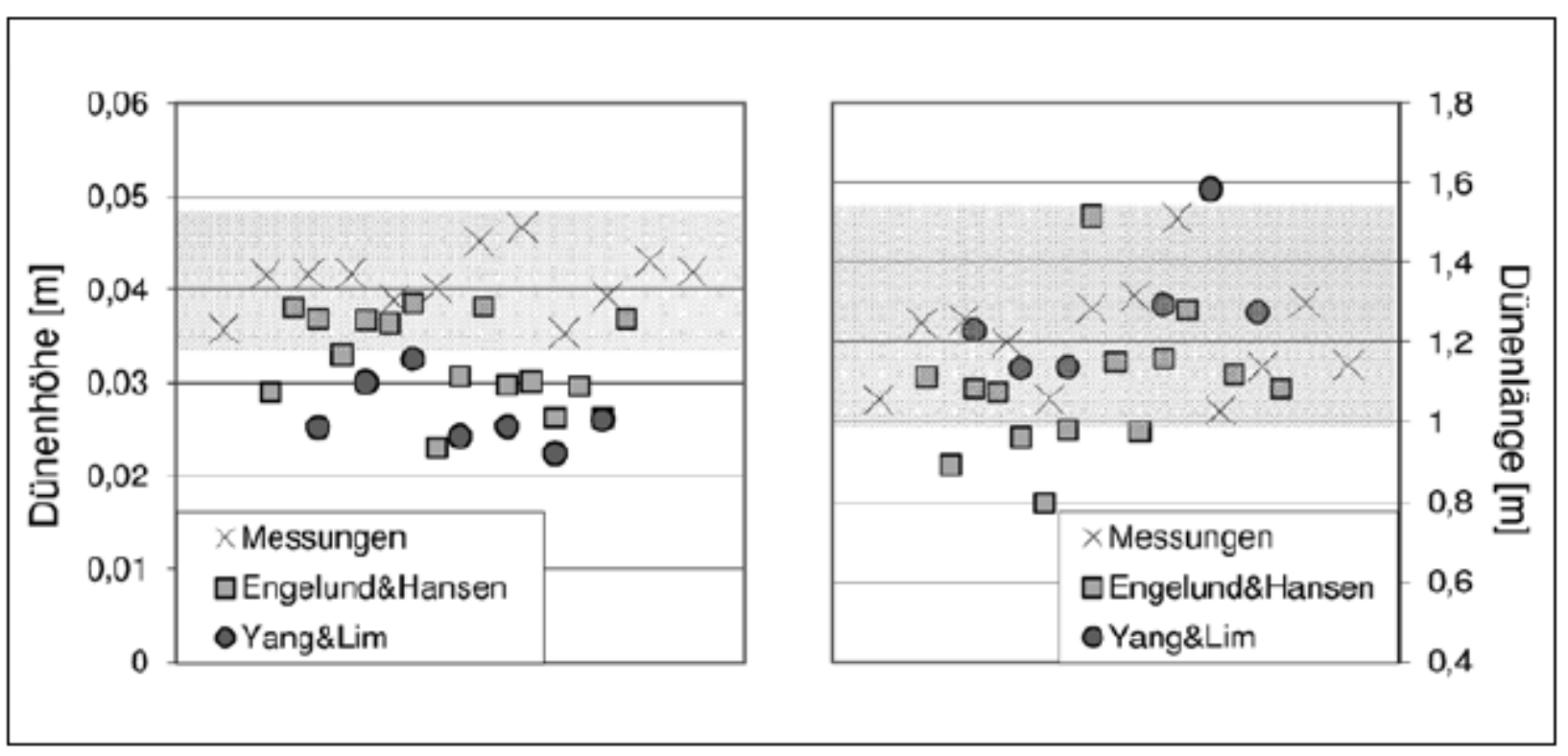

Bild 5: Dünenhöhen und -längen ausgewählter Simulationsläufe mit zwei Transportformeln im Vergleich zu Messungen (Kreuze)

und Smagorinsky-Modell) und zwei Zwei-Gleichungs-Modelle (k- $\varepsilon$ und k- $\omega$ ). Sisyphe löst die ExnerGleichung, wobei neun verschiedene Transportformeln für reinen Geschiebe-, Schwebstoff- und Gesamttransport zur Auswahl stehen. Zusätzlich wurde eine weitere Geschiebetransportformel nach Yang \& Lim [8] implementiert. Der gravitationelle Transport ändert sowohl den Betrag als auch den Transportwinkel und fließt damit ebenfalls in die Transportberechnung ein. Auch hier wurde im Rahmen der hier vorgestellten Studie eine zusätzliche Formel nach Apsley \& Stansby [9] implementiert.

\subsection{Ergebnisse}

Die Bewertung der Qualität der numerischen Ergebnisse findet auf der Basis vornehmlich zweier Parameter statt: Dünenlänge und Dünenhöhe. Diese Parameter sind seit Jahrzehnten herangezogene Größen bei der Betrachtung von Dünen und ihrer Formeigenschaften.

Innerhalb der Parameterstudiewurden Transportformel, Formeln für den gravitationellen Transport, Hiding-and-Exposure-Faktor, Turbulenzmodell, Advektionsschema, Active-Layer-Thickness, Geschiebezugabe(menge), Zeitschritt und Netzauflösung variiert. In diesem Prozess wurde eine weitgehende Netzunabhängigkeit festgestellt und mit einem angepassten Zeitschritt gerechnet 


\section{Autorenfassung}

Goll, Kopmann, Baron: Kontinuierliche Validierung hydrodynamischer und morphodynamischer Modelle mit physikalischen Modellversuchen, 2013

(Courant-Nummer $<1$ ). Die morphodynamische Simulation erwies sich als sensitiv gegenüber Turbulenzmodell und Advektionsschema. Generell kann geschlussfolgert werden, dass mindestens ein Zwei-Gleichungs-Turbulenzmodell für die Transportkörpermodellierung nötig ist, da nur so die Rückströmzonen im Leebereich der Dünen berücksichtigt werden können. Die Advektionsschemata beeinflussen ebenfalls die Strömungsverteilungund liefern so unterschiedliche Ergebnisse. Zusätzlich sind Transportformel und die Transportrichtung ändernden Formeln auschlaggebend für Form, Höhe und Länge der Dünen.

Bild 5 zeigt exemplarisch die Ergebnisse von Dünenhöhen und -längenaus Parametervariation mit den Transportformeln von Engelund \& Hansen und Yang \& Lim. Die Kreuze markieren die Ergebnisse mehrerer physikalischer Modellversuchsreihen. Es wird deutlich, dass sowohl im physikalischen als auch im numerischen Modell eine Streuung der Ergebnisse vorliegt. Tendenziell sind die Werte der Dünenhöhen etwas zu niedrig, während der Bereich der Dünenlänge in einem Großteil der Simulationen getroffen wird. Der Grund für die mangelhafte Abbildung der Dünenhöhen liegt in den fehlenden Dünentälern: Die Dünenkuppen werden gut abgebildet, unzureichend werden die Eintiefungen im Leebreich berechnet. Daher liegteine Möglichkeit zur Optimierung im Bereich der Kalibrierungder Turbulenz (-modelle) und der besseren Abbildungder Strömung im Dünental. Des Weiteren sind Unterschiede je verwendeter Transportformel offensichtlich (Bild 6). Folglich bietet eine Optimierung der verwendeten Transportformel je nach Ausprägung der Dünen oder die Implementierung weiterer Transportformeln einen viel versprechenden Ansatz.

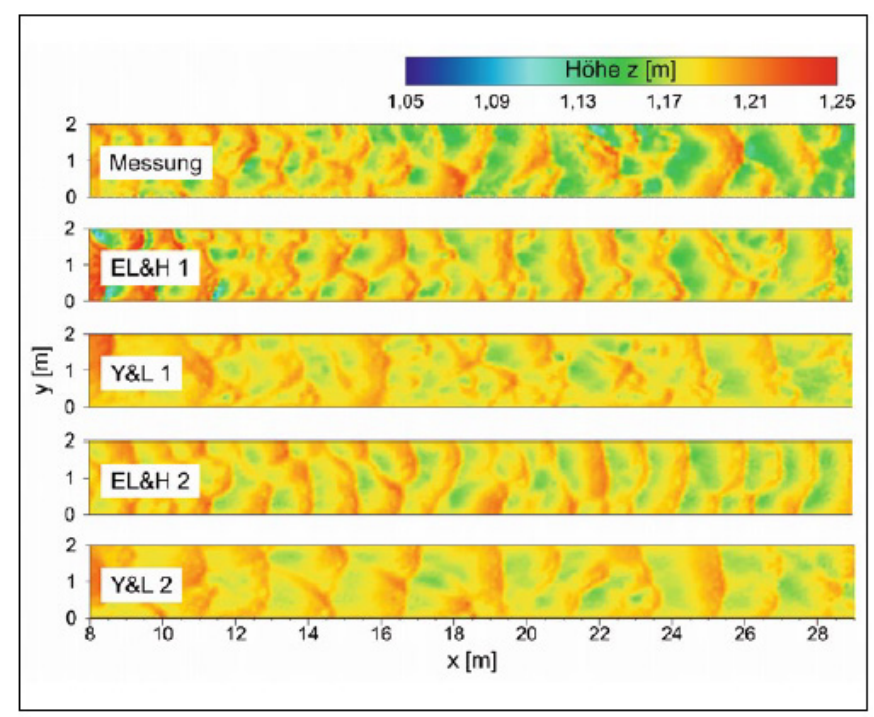

Bild 6: $\quad$ Messung (oben) im Vergleich zu numerischen Simulationen mit Transportformeln von Engelund \& Hansen (EL\&H) und Yang \& Lim (Y\&L): 1) Gravitationeller Transportmit Größenänderung nach Koch \& Flokstra und Winkeländerung nach Apsley \& Stansby; 2) Gravitationeller Transportmit Größenänderung nach Soulsby und Winkeländerung nach Talmon 


\section{Autorenfassung}

Goll, Kopmann, Baron: Kontinuierliche Validierung hydrodynamischer und morphodynamischer Modelle mit physikalischen Modellversuchen, 2013

\subsection{Ausblick}

Die numerische Simulation von Dünenformen, ihre Entstehung und Bewegung wurde mit der Kopplung von Telemac-3D und Sisyphe erfolgreich durchgeführt. Die guten Ergebnisse aus der Vali-

Goll, Kopmann, Baron: Kontinuierliche Validierung hydrodynamischer und morphodynamischer Modelle mit physikalischen Modellversuchen. Wasserwirtschaft 12 (2013), S. 28-33.

dierung mittels Ergebnissen aus der Versuchsrinne sollen nun auf ein Beispiel an Bundeswasserstraßen angewandt werden. Damit soll überprüft werden, ob eine Übertragung auf flussbauliche Maßstäbe möglich ist.

\section{Resumee}

Es wurden anhand von zwei Beispielen die Validierung und der damit einhergehende Verbesserungsprozess der Telemac-Suite dargelegt. Mit Hilfe von Rinnenmodellen können einzelne relevante Phänomene, wie die Wirkung von Buhnen oder Dünen, isoliert betrachtet werden.

Während die Validierungen in dem hydrodynamischen Beispiel dazu dienten, eine optimale Parameterkombination für Prognoserechnungen von Buhnenvarianten abzuleiten, führten die Validierungen im zweiten Beispiel tatsächlich zu Weiterentwicklungen in Sisyphe.

Von der schnellen Entwicklung im Bereich der Messtechnik in den letzten Jahrzehnten profitiert diese Art der Validierung ganz erheblich. War man früher auf einzelne wenige Messpunkte angewiesen, können inzwischen flächig Wasserspiegel, Oberflächengeschwindigkeiten und sogar Sohlenänderungen aufgezeichnet werden. Die enge Verzahnung in der BAW zwischen Rinnenversuchen und numerischer Modellierung zeigt deutliche Synergien. Eine gemeinsame Interpretation von Mess- und Simulationsergebnissen ermöglicht einen erheblichen Erkenntnisgewinn und führt oft zu neuen Messkampagnen sowie neuen Simulationsreihen. 


\section{Autorenfassung}

Goll, Kopmann, Baron: Kontinuierliche Validierung hydrodynamischer und morphodynamischer Modelle mit physikalischen Modellversuchen, 2013

Annalena Goll, Rebekka Kopmann and Manuela Baron

Continuous Validation of Hydrodynamic and Morphodynamic Models by the Use of Physical Experimental Flumes

On the basis of two examples the validation due to laboratory experiments and the resulting benefit for the Telemac-Suite are presented. By the aid of flume experiments individual relevant phenomenon, as the influence of groynes and dunes, can be considered separately, analysed and evaluated. Two flume experiments and their simulation with the Telemac-Suite are described: One hydrodynamic experiment with fixed bed to determine the optimal parameter combination for prognosis calculations of groyne variations, as well as a morphodynamic experiment which deals with the emergence and movement of dunes. The latter belongs into the field of research and serves the further development of morphodynamic flume experiments as well as numerical simulations. In contrast to this, the former hydrodynamic groyne experiment is in step with actual practice.

Анналена Голл, Ребекка Копманн и Мануэла Барон

Непрерывная валидация гидродинамических и морфодинамических моделей с физическим экспериментальным моделированием

На основе двух примеров представлена валидация посредством лабораторных испытаний и последующая польза применения для программного обеспечения TelemacSuite. C помощью желобных моделей и их цифрового моделирования может быть осуществлено изолированное наблюдение, анализ и оценка отдельных релевантных феноменов, таких как эффект действия волнорезов или дюн. Представлен гидродинамический эксперимент с использованием желобов с твердой подошвой для определения оптимальной комбинации параметров для прогностического расчета вариантов волнорезов, а также морфодинамические опыты для исследования образования и движения дюн.

\section{Danksagung}

Die Autoren danken Petra Faulhaber, Martin Henning und Thorsten Hüsener für die Bereitstellung der Messdaten, Diskussionen und die gute Zusammenarbeit 


\section{Autorenfassung}

Goll, Kopmann, Baron: Kontinuierliche Validierung hydrodynamischer und morphodynamischer Modelle mit physikalischen Modellversuchen, 2013

\section{Autoren}

Dipl.-Ing. Annalena Goll

Dipl.-Ing. Rebekka Kopmann

Bundesanstalt für Wasserbau

Kußmaulstr. 17

76185 Karlsruhe

Annalena.goll@baw.de

Rebekka.kopmann@baw.de

\section{Dipl.-Ing. Manuela Baron}

Ingenieurbüro Baron

Vorholzstr. 50

76137 Karlsruhe

Manuela.baron@web.de

\section{Literatur}

[1] Hervouet, J.-M: Hydrodynamics of free surface flows: modelling with the finite element method. Chichester: Wiley (Formerly CIP), 2007.

[2] Henning, M.; Hentschel, B.; Hüsener, T.: Photogrammetric system for measurement and analysis of dune movement. In: Proceedings of 33rd IAHR Congress: Water Engineering for a Sustainable Environment (2009), S. 4 965-4 972.

[3] Hüsener, T.; Faulhaber, P.; Baron, M.: Modifikationen in bestehenden Stromregelungssystemen an Wasserstraßen Untersuchung mit gegenständlichen und numerischen Verfahren. In: Wasserbau Symposium 2012 „Wasser-Energie, Global denken-lokal handeln“, Graz, Österreich, 2012.

[4] Baron, M.: Kalibrierung und Validierung der numerischen Verfahren Telemac und UnTRIM anhand einer Laborrinne. Berichtzum BAW-Auftrag „Begleitendemehrdimensionale numerische Modellierung von Rinnenversuchen“, 2013.

[5] Villaret, C.; Hervouet, J.-M.; Kopmann, R.; Merkel, U. H.; Davies, A.G.: Morphodynamic modeling using the telemac finite-element system. In: Computers \& Geosciences (2011), Heft 53, S. 105-113.

[6] Maerker, C.; Malcherek, A.: Die Analyse von Baggern und Verklappen. Teil 1: Das Softwarepaket DREDGESIM. In: Korrespondenz Wasserwirtschaft (2010), Heft 10, S. 550-555. 


\section{Autorenfassung}

Goll, Kopmann, Baron: Kontinuierliche Validierung hydrodynamischer und morphodynamischer Modelle mit physikalischen Modellversuchen, 2013

[7] Goll, A.; Kopmann, R.: Dune simulation with TELEMAC-3D and SISYPHE: A parameter study. In: Proceedings of the XIXth TELEMAC-MASCARET User Conference (2012), S. 19-25.

[8] Yang, S.-Q.; Lim, S.-Y.: Total load transport formula for flow in alluvial channels. In: Journal of Hydraulic Engineering (2003), Heft 128, S. 68-72.

[9] Apsley, D. D.; Stansby, P. K.: Bed-load sediment transporton large slopes: Model formulation and implementation within a RANS solver. In: Journal of Hydraulic Engineering (2008), Heft 134, S. 1 440-1 451. 